\title{
DYNAMICAL INSTABILITY OF THE ENVELOPES OF RED SUPERGIANTS AND THE ORIGIN OF PLANETARY NEBULAE
}

\author{
B. PACZYŃSKI and J. ZıóŁKOWSKI \\ (Institute of Astronomy, Polish Academy of Sciences, Poland)
}

\begin{abstract}
A BSTRACT
Deep convective envelopes of the red supergiants with masses smaller than $4 M_{\odot}$ are found to be dynamically unstable for the luminosities in the range of $4 \times 10^{3}-5 \times 10^{4} L$. The total energy of the envelopes is positive when the recombination energy of the ionized hydrogen and helium is taken into account, and is sufficient to expel a typical envelope up to infinite distance at a speed of $30 \mathrm{~km} / \mathrm{sec}$. We suggest that planetary nebulae are formed in this way.
\end{abstract}

Substantial progress has been recently achieved in the observational approach to the evolution of the planetary nebulae and their nuclei (O'Dell, 1963; Seaton, 1966). A number of theoretical papers have been devoted to the interpretation of the central stars of planetary nebulae in terms of stellar models composed of helium and heavier elements (L'Ecuyer, 1966; Rose, 1966, 1967; Vila, 1966). The evolutionary tracks and the time-scales obtained by Vila (1966) and Rose (1967) show the closest similarity to the empirical track of Seaton (1966). These theoretical sequences were obtained for stellar models of $0 \cdot 75-1 \cdot 2 M_{\odot}$, cooling down towards the white-dwarf region. There is a widespread opinion that planetary nebulae originate from red giants. This idea was suggested by Shklovsky (1956), and recently Abell and Goldreich (1966) have summarized a series of arguments that tend to support this point of view. According to Osterbrock (1964) there was no satisfactory identification of the physical processes involved in the formation of the planetary nebulae. Recently, Rose $(1966,1967)$ suggested that thermal and pulsational instabilities found by him in the models of helium shell-burning stars may be connected with the origin of the planetary nebulae.

We found that red supergiants of sufficiently high luminosity have extended convective envelopes that are dynamically unstable. This phenomenon is due to a low value of the adiabatic exponent $\Gamma_{1}$ in the thick zones of partial ionization of hydrogen and helium. This dynamical instability is of the same character as the one encountered in the pre-main-sequence stars just before they reach the Hayashi track. There are two main differences between an old supergiant and a young proto-star: a red supergiant has a very dense core composed of helium and heavier elements and it evolves upwards on the H-R diagram towards the border of dynamical stability. It is not clear whether or not a helium shell-burning star may lose the hydrogen-rich envelope as a result of thermal or pulsational instabilities associated with the shell sources. If not,

Osterbrock and O'Dell (eds.), Planetary Nebulae, 396 399. I.A.U. 
then it seems that nuclear evolution of a single star may be terminated either as a result of the dynamical instability of the core, or because of the dynamical instability of the envelope. The first case was discussed by many authors as a likely cause of supernova explosions (Hoyle and Fowler, 1960; Colgate and White, 1966). The second will be discussed in this paper.

To construct static envelopes the computer program written by Ziółkowski (1967) was used. All the calculations were carried out with the GIER computer of the Warsaw University. Our program was almost identical with the one described by Baker and Kippenhahn (1962) and Hofmeister et al. (1964). There were only two minor differences: the dependence of the temperature on the optical thickness in the atmosphere was, in the present study, the same as that adopted by Henyey et al. (1965, eq. 49); and the opacities published by Cox and Stewart (1965) were used in our program. Model envelopes were integrated until the temperature increased to $5 \times 10^{5}{ }^{\circ} \mathrm{K}$. We adopted the ratio of mixing length to pressure scale height, $l / H_{\mathrm{p}}=1$. The luminosity $L$, and the chemical composition were assumed to be constant in a given model. Because of the high luminosities and the low effective temperatures which were of interest here the convection was strongly superadiabatic and the density changed very little in a typical envelope. The transition between the extended envelope and a small and dense core was very sharp, and in most cases occurred at fractions of the radius $(0 \cdot 0005-0 \cdot 01)$.

The eigenvalue $\sigma_{0}^{2}$ corresponding to the fundamental mode of the adiabatic radial oscillations was computed for every static envelope. For a given total mass $M$ of a star, and an effective temperature $T_{\mathrm{e}}$, we searched for the luminosity $L$ at which $\sigma_{0}^{2}=0$. As a by-product the mass fraction contained in a dense core was obtained for such a model. In this way it was possible to find a line on the $M_{\mathrm{bol}}-\log T_{\mathrm{e}}$ diagram which separated the stable and the unstable static envelopes. The unstable envelopes were, of course, found on the high-luminosity side of that line.

The total energy of an envelope that became unstable was positive when the recombination energy of the ionized hydrogen and helium was taken into account. Therefore, it is likely that instability may cause an expansion of the envelope to infinity. If the envelope expanded adiabatically it would attain a velocity of the order of $30 \mathrm{~km} / \mathrm{sec}$ in a typical case. This is remarkably close to the expansion velocities observed in planetary nebulae.

The borderlines on the $M_{\mathrm{bol}}-\log T_{\mathrm{e}}$ diagram were obtained for dynamically unstable envelopes of the population-I stars of 1,2 , and $3 M_{\odot}$, and for the population-II star of $1 M_{\odot}$. The Kippenhahn I and II mixtures (Cox and Stewart, 1965) were used for the population I and II, respectively. The borderlines are shown in Figure 1 for the population-I stars. The dynamical instability appears for the population-II star at a bolometric magnitude $1^{\mathrm{m}}$ above the line for $1 M_{\odot}$ star of population $\mathrm{I}$. At the highest luminosities allowed by the assumptions that stellar models are in hydrostatic and thermal equilibrium the envelopes of stars of 5 and $15 M_{\odot}$ were dynamically stable. 


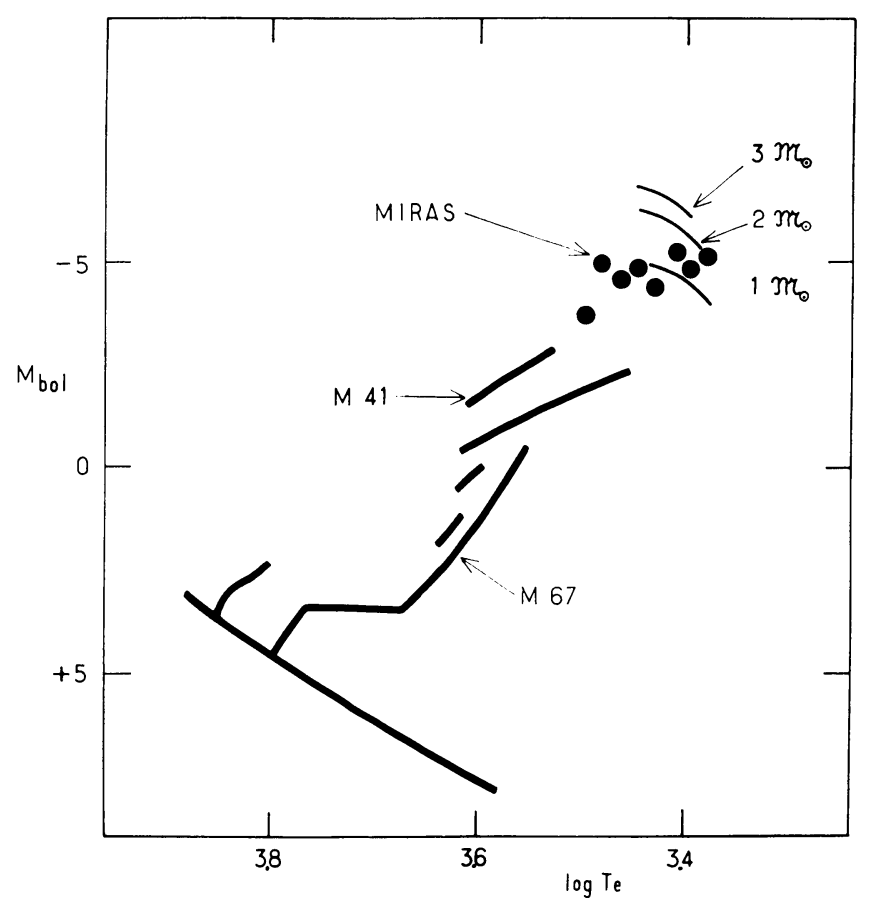

FIG. 1. The borderlines for dynamically unstable envelopes of population-I stars of $I, 2$, and $3 M_{\odot}$ are shown on the $M_{\mathrm{bol}}-\log T_{\mathrm{e}}$ diagram. The mass fraction contained in the dense cores of the stars varies from 0.2 to 0.8 between the low-and high-temperature ends of each line. Positions of some galactic clusters are adopted from Sandage (1957) and of the Mira variables from Smak (1966).

We propose the following scheme for the late phases of stellar evolution. After the exhaustion of helium in the core the star evolves into the region of red supergiants and moves up on the H-R diagram very close to the Hayashi border (see e.g. Kippenhahn et al., 1965; Weigert, 1966; Sugimoto and Yamamoto, 1966). The mass of the helium and carbon core and the luminosity due to the helium and hydrogen-shell sources increase. A star with total mass smaller than about $4 M_{\odot}$ will terminate this type of evolution with an outflow of hydrogen-rich matter as a result of the dynamical instability of the extended envelope. We suggest that planetary nebulae are formed in this way. This process seems to be impossible for a star with larger mass. In the latter case the mass of the core in which all the nuclear-energy sources are exhausted will finally exceed the Chandrasekhar limit for degenerate configurations. Dynamical instability of the core, followed by the supernova explosion, may be expected.

It transpires from Figure 1 that the region in the H-R diagram in which stellar envelopes might be dynamically unstable is occupied by Mira-type stars at maximum light (Smak, 1966). This may suggest that there is some connection between our 
instabilities and the light changes in the long-period variables, and also that those stars might be the direct ancestors of the planetary nebulae.

We would like to point out that stellar models with the highest luminosity obtained by Sugimoto and Yamamoto (1966) for $1 M_{\odot}$ should have dynamically unstable envelopes. The dense core of $0.8 M_{\odot}$ obtained by Weigert (1966) at the end of his evolutionary track for $5 M_{\odot}$ had a luminosity sufficiently high to induce instability of a hydrogen-rich envelope of $0.8 M_{\odot}$. Therefore, some evolutionary tracks already available approach the phase in which a star may lose its outer layers and form a planetary nebula.

The numerical values of the luminosities and masses of stars with dynamically unstable envelopes are highly uncertain because of the poorly known theory of convection, opacities at low temperatures, and the boundary conditions for the stability tests. More detailed description of our work and more complete discussions of the results obtained will be published soon in Acta Astronomica.

\section{References}

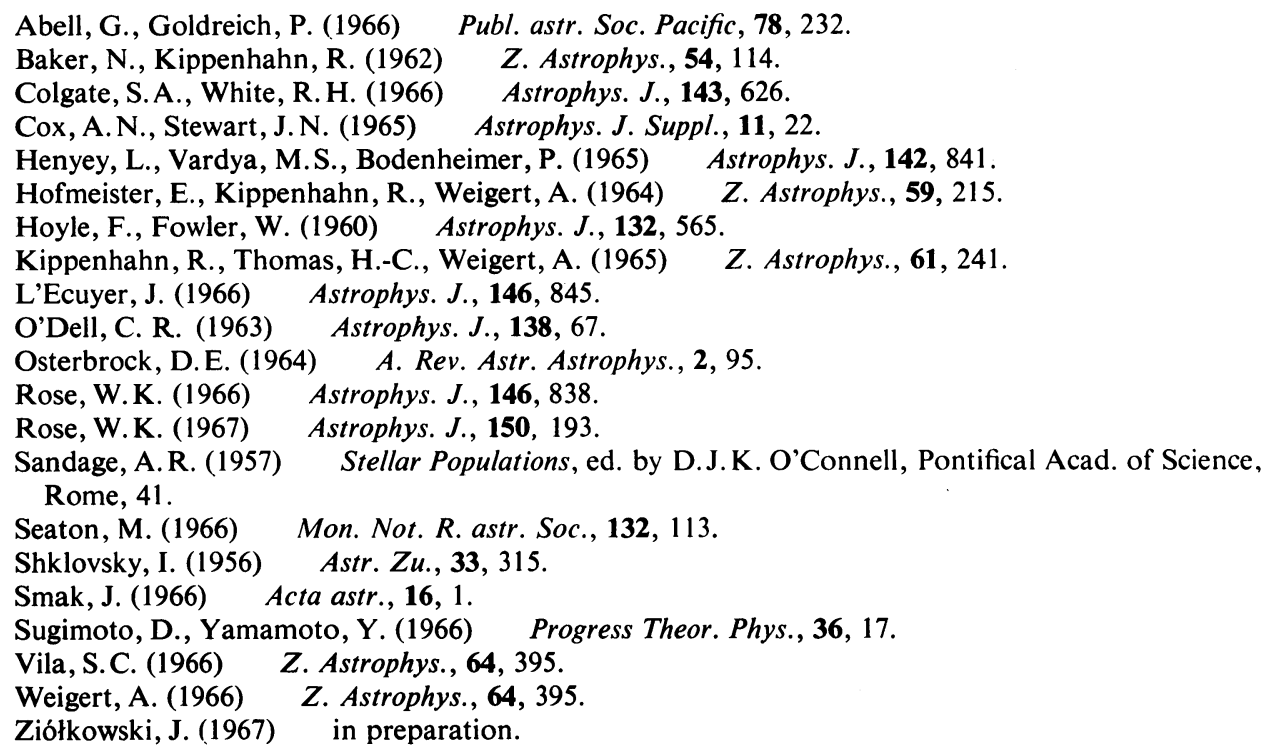

